\title{
Subwavelength Hyperlens Resolution With Perfect Contrast Function
}

\author{
Novitsky, Andrey; Repän, Taavi; Zhukovsky, Sergei; Lavrinenko, Andrei
}

Published in:

Annalen der Physik

Link to article, DOI:

10.1002/andp.201700300

Publication date:

2018

Document Version

Version created as part of publication process; publisher's layout; not normally made publicly available

Link back to DTU Orbit

Citation (APA):

Novitsky, A., Repän, T., Zhukovsky, S., \& Lavrinenko, A. (2018). Subwavelength Hyperlens Resolution With

Perfect Contrast Function. Annalen der Physik, 530(3), [1700300]. https://doi.org/10.1002/andp.201700300

\section{General rights}

Copyright and moral rights for the publications made accessible in the public portal are retained by the authors and/or other copyright owners and it is a condition of accessing publications that users recognise and abide by the legal requirements associated with these rights.

- Users may download and print one copy of any publication from the public portal for the purpose of private study or research.

- You may not further distribute the material or use it for any profit-making activity or commercial gain

- You may freely distribute the URL identifying the publication in the public portal 


\title{
Subwavelength Hyperlens Resolution With Perfect Contrast Function
}

\author{
Andrey Novitsky,* Taavi Repän, Sergei V. Zhukovsky, and Andrei V. Lavrinenko
}

Recently it has been shown that plasmonic effects in hyperbolic metamaterials may facilitate overcoming the diffraction limit and enhance the contrast function of an image by filtering background radiation. Unfortunately, the contrast function of such a dark-field hyperlens degrades in the deep-subwavelength regime. We push forward the concept of the contrast function revival in the subwavelength imaging by introduction of the proper phase difference between coherent sources. To study this effect we develop a simplified theory of the wave propagation through a hyperbolic metamaterial and show that, in principle, two sources standing apart at any subwavelength distance can be distinguished. We suggest two feasible designs, the first of which employs the obliquely incident light, while the second one is based on a properly designed metasurface. The concept can be used in high-contrast subwavelength microscopy.

\section{Introduction}

The resolution of the optical imaging systems is known to be Abbe diffraction limited with roughly a half of the wavelength..$^{[1]}$ This fundamental limit was beaten using the negative-refractiveindex superlenses, ${ }^{[2]}$ which transmit both propagating and evanescent waves. The resolution was improved due to preserving of evanescent waves bearing the information about the subwavelength details typically lost in conventional optical systems. Another issue to be taken into account is the contrast function of the image. Transmitted through the superlens, the weak subwavelength image may be blurred by the strong background radiation. In this case bright-field amplitude microscopy is not appropriate, and phase-contrast microscopy ${ }^{[3]}$ and related techniques (differential interference contrast microscopy, ${ }^{[5,15]}$ Hoffman modulation contrast microscopy, ${ }^{[6]}$ etc.) should be used instead. The optical path differences in phase-contrast microscopes are converted to the observable changes in image brightness. The phasecontrast techniques are widely applied for watching biological specimens without damaging them, ${ }^{[7-10]}$ however, their resolution is diffraction limited.

Dr. A. Novitsky, T. Repän, Dr. S. V. Zhukovsky, Dr. A. V. Lavrinenko DTU Fotonik

Technical University of Denmark

Ørsteds Plads 343, DK-2800 Kongens Lyngby, Denmark

E-mail:anov@fotonik.dtu.org

The ORCID identification number(s) for the author(s) of this article can be found under https://doi.org/10.1002/andp.201700300

DOI: $10.1002 /$ andp. 201700300
Another technique to achieve the high contrast and simultaneously break the diffraction limit is the dark-field hyperlensing. A hyperlens itself is a slab of hyperbolic metamaterial (HMM) designed as a planar/cylindrical/spherical structure transmitting plane waves with large wavenumbers $k^{[11-14]}$ The high- $k$ waves (as they are called in this case) generated outside the lens can propagate in HMMs due to the unique hyperbolic isofrequency surfaces characteristic to the metamaterial. ${ }^{[15]}$ HMMs may be designed in the wire- or slab-fashion and possess a number of remarkable properties and promising applications. ${ }^{[16-24]} \mathrm{A}$ planar hyperlens as a stack of periodically arranged metal and dielectric layers is shown in Figure 1a. Transmission of evanescent waves generated by the sources resembles that of a superlens, but in contrast to the latter the hyperlens has more degrees of freedom related to two types of HMMs. A HMM of the first type (HMM-I) transmits the whole spectrum of the waves, that is both propagating and evanescent waves (see the dashed curves in Figure 1b). Background radiation is scattered by an object and they pass together demonstrating low-contrast brightfield microscopy. ${ }^{[25-29]}$ The dispersion equation of the second type HMM (HMM-II) forbids transmission of low- $k$ waves, thus filtering out background radiation completely (solid curves in Figure 1b). Meanwhile, high- $k$ light scattered from the source captures subwavelength features and images with the high contrast. ${ }^{[30]}$ Dark-field hyperlenses based on the HMM-II are considered in this article.

The meaning of the contrast function is to quantify the discernibility of two separate objects in the image plane, the images of the sources being displayed as the peaks separated by the dip (see two such configurations in Figure 1a). Depending on how great is the difference between the intensities of the lower peak $I_{p}$ and $\operatorname{dip} I_{d}$ compared to their aggregated intensity we may judge of the discernibility of the sources using the contrast function

$$
C F=\frac{I_{p}-I_{d}}{I_{p}+I_{d}}
$$

If $C F=1$, the sources are perfectly distinguishable. When the background radiation is negligible as in the case of the darkfield hyperlens, the contrast function can be close to perfect one, $C F=1$. The other limiting case $C F=0$ means that the individual objects cannot be observed. 

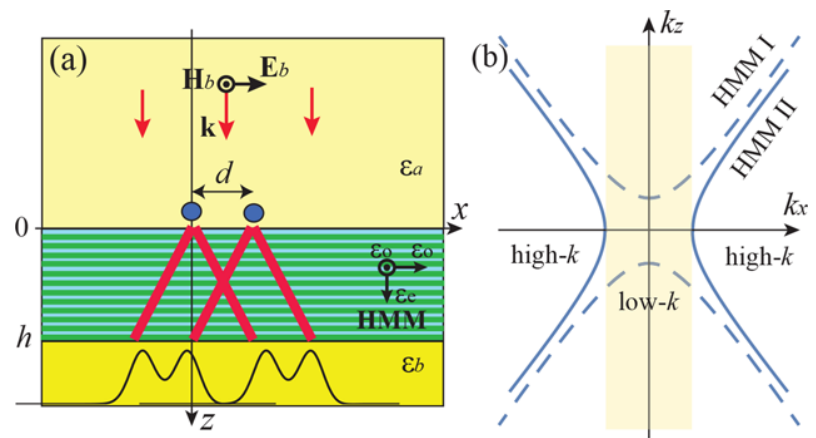

Figure 1. Hyperbolic metamaterial and its distinctive spatial-frequency spectrum. a) Geometry of the dark-field planar hyperlens under consideration: background plane wave excites two electric dipole sources which radiation is transmitted along the red rays in HMMs. Output is a couple signals on the right- and left-hand sides. b) Hyperbolic isofrequency curves of HMM-I and HMM-II. HMM-II supports only high-k waves.
As demonstration of the issues discussed in the present article, we consider a cylindrical dark-field hyperlens formed by the periodically arranged metallic and dielectric cylindrical layers (see Figure 2a). Two sources are located in the vicinity of the inner interface of the lens. They can be resolved by a conventional optical setup if the distance between them is greater than $\lambda / 2 \mathrm{NA}$, where $\lambda$ is the wavelength and NA is the numerical aperture. Assuming the maximum value of the numerical aperture in air $\mathrm{NA}=1$, one concludes that the sources cannot be distinguished, when $d<\lambda / 2$. As well as a planar hyperlens, the cylindrical darkfield hyperlens in Figure 2a transmits the field in the form of two pairs of peaks, each pair being formed by both sources. If the peaks are distinguished, the contrast function is high. The subwavelength imaging capability of the cylindrical hyperlens is inspected in Figure $2 \mathrm{~b}$. When the sources are spaced by more than a half-wavelength $(d>350 \mathrm{~nm})$, they can be quite well distinguished, albeit for $d>500 \mathrm{~nm}$ the two pairs of images start to overlap. In the near-subwavelength regime $(d=250-350 \mathrm{~nm})$,
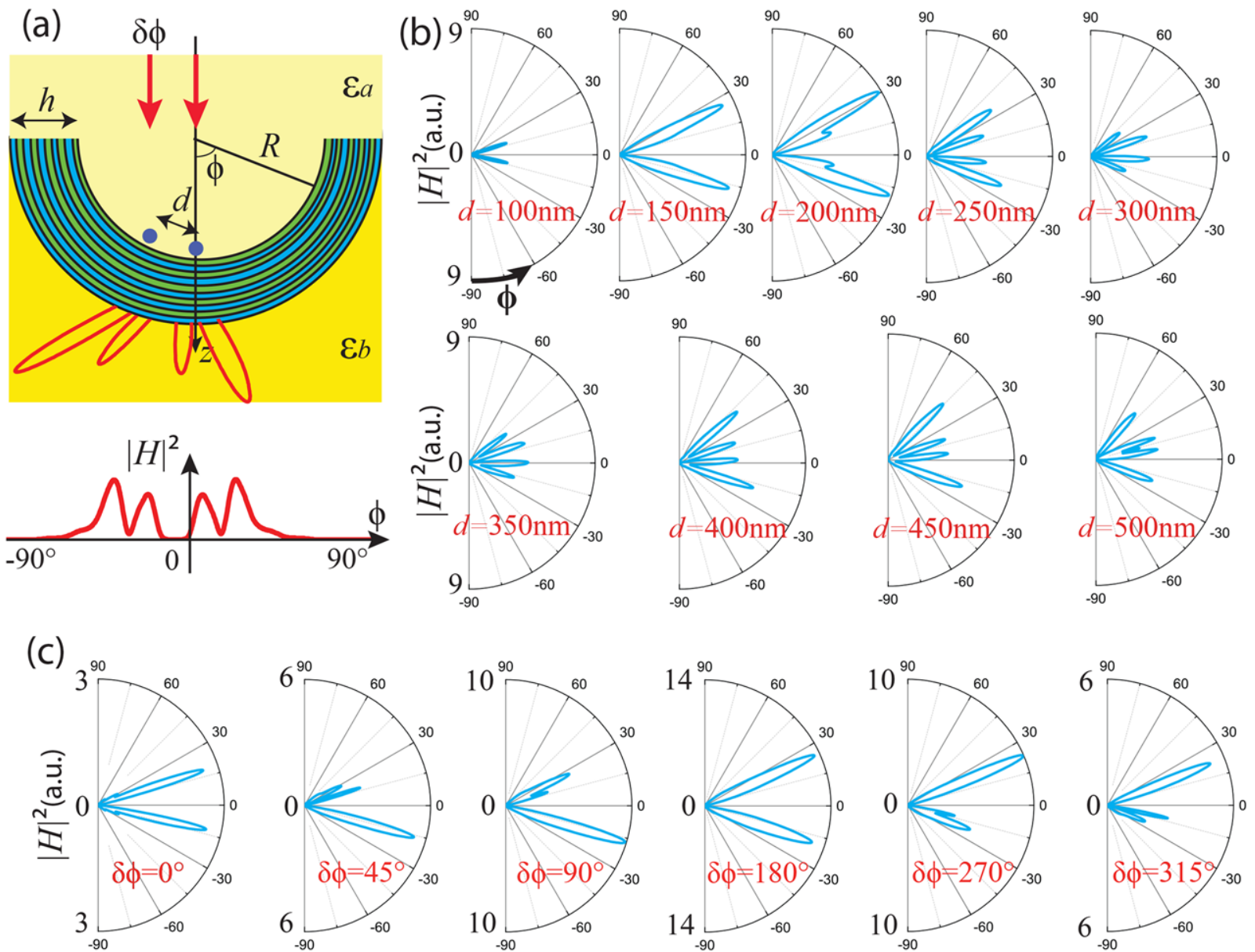

Figure 2. Intensity $|H|^{2}$ of the field of background and two dipoles transmitted through the cylindrical hyperlens. a) Sketch of the cylindrical hyperlens. Radiation of two dipoles results in the distribution over the polar angles $\phi$. Example of intensity collected at the outer radius of the lens $R+h$ is shown at the bottom. Transmitted intensity $|H(\phi)|^{2}$ for b) different distances $d$ between the particles (phase difference $\left.\delta \phi=0\right)$ and c) different phase differences $\delta \phi$ (distance $d=100 \mathrm{~nm}$ ). Parameters as in Ref. [30]: wavelength $\lambda=715 \mathrm{~nm}$, radius of spherical particle $R_{p}=35 \mathrm{~nm}$, permittivity of particle $\varepsilon_{p}=2$, distance from the particle to $\mathrm{HMM} u=R_{p}$, permittivities of the input $\varepsilon_{a}=1$ and output $\varepsilon_{b}=3^{2}$ media, inner radius $R=1000 \mathrm{~nm}$, metal $\varepsilon_{m}=(0.14+i 2.26)^{2}$ and dielectric $\varepsilon_{m}=1.45^{2}$ permittivities, metal $h_{m}=15 \mathrm{~nm}$ and dielectric $h_{d}=15 \mathrm{~nm}$ thicknesses, and number of unit cells $N=20$ (effective permittivities of $\mathrm{HMM} \varepsilon_{0}=-1.49+0.316 i$ and $\varepsilon_{e}=7.04+0.601 i$, its thickness $h=600 \mathrm{~nm}$ ). Background field is azimuthally homogeneous (see Section S2 in Supporting Information for details). 
the objects are also perfectly resolved in the image plane. However, the deep-subwavelength features (the separation distance of $200 \mathrm{~nm}$ and less) appear to be not resolved. Similar behavior was mentioned in Ref. [30] Thus, the resolution (contrast function) naturally degrades with the decrease of distance $d$ between the sources/objects.

In this paper, we reveal the technique of revival of contrast function for objects separated by subwavelength distances $d$ based on the imposing of extra phase difference $\delta \phi$ between the sources. Let us look at the effect brought by the phase acquired by one of the objects as shown in Figure 2a. The phase difference tremendously changes both the magnitude of the transmitted radiation and its contrast (see Figure 2c). The $\pi$-shift $\left(\delta \phi=180^{\circ}\right)$ enhances the transmission in more than 4 times. What is more curious, the resolution of two sources is again quite good at $\delta \phi=$ $45^{\circ}$ by sacrifice the symmetry of the pattern. Asymmetry is apparently related to the phase difference. The deep-subwavelength object $(\lambda / 7)$ can be distinguished in some range of phases $\delta \phi$ (look at the case of $\delta \phi=90^{\circ}$ ). The transmitted pattern flips, if $\delta \phi \rightarrow-\delta \phi$, and two distinct peaks can be detected in another region of the cylindrical hyperlens.

This article is devoted to physics behind the revival of the contrast function of the dark-field hyperlens. We show that the contrast function can be made perfect (equal to 1) independently from the distance between two objects. Interestingly, improvement of the lens contrast has the same roots as the phase-shift mask technique applied in lithography, ${ }^{[31]}$ because the operational principle of both approaches is grounded on the destructive interference of coherent signals. Moreover, this effect could be expected, because there is a prior knowledge (coherence and phase shift) which "reduces the set of possible configurations and thereby improves the resolution". ${ }^{32]}$

The article consists of three more sections. Section 2 delivers the theoretical background for the improvement of the contrast function with the help of the phase shift between two sources. This simplified theory serves as a basis for closed-form modeling of realistic systems. We discuss two methods to impose a phase difference, with the oblique incidence of plane waves and metasurface on top, in Section 3. Outlook of the contrast function for multiple objects at subwavelength distances (realistic imaging) and conclusion can be found in Section 4.

\section{Calculation and Simplified Theory}

In this section we elaborate a theory of the light transmission through the type-II HMM planar slab in application to the effect of the contrast function revival by means of the introduced phase difference. The ultimate analytical expressions are very simple and include four parameters that can be found either from simulations or in experiment.

We consider geometry demonstrated in Figure 1a: a type-II HMM slab is sandwiched between two half-infinite dielectric media with dielectric permittivities $\varepsilon_{a}$ and $\varepsilon_{b}$. A monochromatic background plane wave is normally incident from medium $a$ exciting two electric dipoles at distance $d$ one from another. The dipoles are mimicked by identical spherical particles of radius $R_{p}$ and permittivity $\varepsilon_{p}\left(R_{p}=35 \mathrm{~nm}\right.$ and $\varepsilon_{p}=2$ except Figure 5$)$. The dipoles lie on the hyperlens, hence, the distance from particle's center to the interface equals $u=R_{p}$. Dipole's polarizability is of the form $\alpha_{p}=R_{p}^{3}\left(\varepsilon_{p}-\varepsilon_{a}\right) /\left(\varepsilon_{p}+2 \varepsilon_{a}\right)$, while its magnetic field equals

$\mathbf{H}_{d}=k_{0}^{2} \sqrt{\varepsilon_{a}} \frac{\mathbf{r} \times \mathbf{p}}{r^{2}}\left(1+\frac{i}{\sqrt{\varepsilon_{a}} k_{0} r}\right) \mathrm{e}^{i \sqrt{\varepsilon_{a}} k_{0} r}$,

where $\mathbf{r}$ is the radius-vector from the dipole to the observation point, $\mathbf{p}=\alpha_{p} \mathbf{E}_{p}$ is the dipole moment, and $\mathbf{E}_{p}$ is the electric field at the center of the sphere.

We aim to describe transmission of radiation emitted by these dipoles in a simplified closed-form manner. The planar HMM slab consists of $N$ periods of alternating metal and dielectric layers with permittivities and thicknesses respectively $\varepsilon_{m}, h_{m}$ and $\varepsilon_{d}$, $h_{d}$. We rely on the effective medium theory ${ }^{[16]}$ valid for subwavelength periods $h_{m}+h_{d} \ll \lambda$, within which the HMM is characterized by the uniaxial effective permittivity tensor $\hat{\varepsilon}=\left(\varepsilon_{0}, \varepsilon_{0}, \varepsilon_{e}\right)$ and slab thickness $h=N\left(h_{m}+h_{d}\right)$, where ordinary permittivity $\varepsilon_{o}=\frac{\varepsilon_{m} h_{m}+\varepsilon_{d} h_{d}}{h_{m}+h_{d}}$ and extraordinary permittivity $\varepsilon_{e}=\frac{\varepsilon_{m} \varepsilon_{d}\left(h_{m}+h_{d}\right)}{\varepsilon_{m} h_{d}+\varepsilon_{d} h_{m}}$ (see Figure 1a). HMM-II behaves as a metal in the $x-\gamma$ plane and has the negative ordinary permittivity $\varepsilon_{o}^{\prime}<0$. At the same time, it possesses the properties of a dielectric along the $z$ axis having the positive extraordinary permittivity $\varepsilon_{e}^{\prime}>0$. Here and below $\varepsilon^{\prime}$ and $\varepsilon^{\prime \prime}$ stand for the real and imaginary parts of the permittivity. The hyperbolic character of the dispersion law is clearly seen from the dispersion equation for the extraordinary wave $k_{z}^{2} / \varepsilon_{o}+k_{x}^{2} / \varepsilon_{e}=(\omega / c)^{2}$ at $\varepsilon_{o}^{\prime} \varepsilon_{e}^{\prime}<0$, where $\omega$ is the circular frequency and $c$ is the speed of light. It is important that owing to the negative sign of ordinary permittivity $\varepsilon_{0}^{\prime}$ the type-II HMMs do not support propagation of the low- $k$ waves.

To excite extraordinary waves in the hyperbolic metamaterial we employ a background plane wave, which magnetic field is $\gamma$-directed as $\mathbf{H}_{b}=H_{b} \mathbf{e}_{\gamma}$. Electric field $\mathbf{E}_{p}$ in the point of location of the dipole particles (sources) is the sum of the background and scattered fields. The latter is the superposition of the field reflected from the HMM slab and the field radiated by neighboring dipole. Assuming the dipoles are quite distant, we neglect the dipole-dipole interaction. As demonstrated in Section S1.3 of Supporting Information, this approximation works well for the distances between particles down to $100 \mathrm{~nm}$, because the HMM-particle interaction prevails owing to the shorter distance between them (it is equal to the radius $R=35 \mathrm{~nm}$ ). Thus, $\mathbf{E}_{p} \approx E_{p} \mathbf{e}_{x}$ and magnetic field of the dipole $\mathbf{H}_{d}$ is $\gamma$-polarized. Then the magnetic field incident onto the interface between the input medium and HMM equals $\mathbf{H}_{i n c}(x, z=0)=H_{i n c}(x) \mathbf{e}_{y}$ and we can reduce our consideration to the scalar theory with the incident magnetic field $H_{\text {inc }}(x)=H_{b}+H_{d}(x)+H_{d}(x-d)$.

Further we calculate the transmitted magnetic field accurately through the Fourier transform (decomposition over the plane wave spectrum) as follows

$H_{t r}(x)=\int_{-\infty}^{\infty} t\left(k_{x}\right) H_{i n c}\left(k_{x}\right) \mathrm{e}^{i k_{x} x} d k_{x}$

where $k_{x}$ is the transverse wavenumber,

$t\left(k_{x}\right)=\frac{2}{\left(1+\frac{\gamma_{b}}{\gamma_{a}}\right) \cos \left(k_{z} h\right)-i\left(\frac{k_{z}}{k_{0} \gamma_{a} \varepsilon_{0} \varepsilon_{e}}+\frac{\gamma_{b} \varepsilon_{k_{0}} k_{0}}{k_{z}}\right) \sin \left(k_{z} h\right)}$ 
(a)

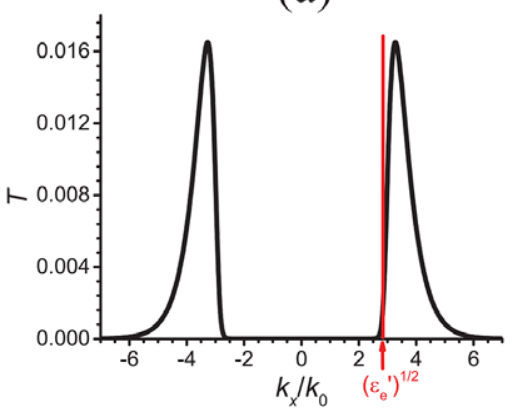

(b)

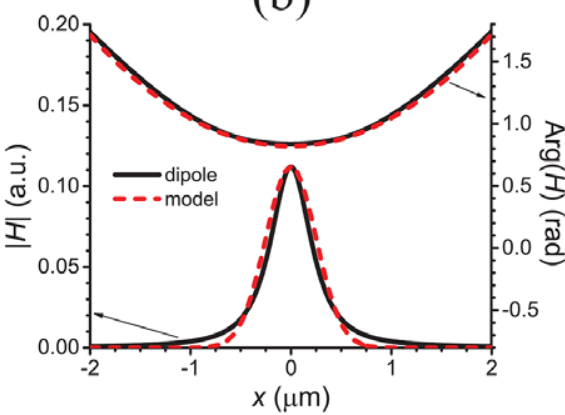

(c)

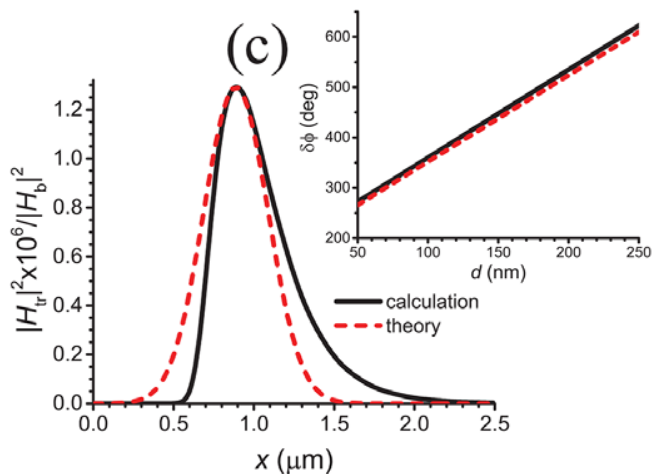

Figure 3. Rationale of the restrictions imposed by the simplified theory of transmission through the HMM-II slab and retrieval of its parameters. a) Transmission (4) through the HMM II slab occurs near $k_{x}= \pm k_{0} \sqrt{\varepsilon_{e}^{\prime}}$. b) $|H|$ and $\operatorname{Arg}(H)$ of the dipole radiation (2) and its Gaussian approximation [half-width $\Delta x=(0.25+0.0035 i) \mu \mathrm{m}, \operatorname{Arg}(H)$ is lifted by 0.82 in the model]. c) Calculated transmitted intensity (3) vs. transverse coordinate $x$ and the line of unit contrast function (in the inset) are shown by solid curves and determine 4 parameters of the simplified theoretical modeling. Intensity profile (7) and dependence $\delta \phi(d)$, Eq. (9), calculated using the simplified theory for the retrieved parameters $a=283.2 \mathrm{~nm}, b^{\prime}=888.7 \mathrm{~nm}, b^{\prime \prime}=421.5 \mathrm{~nm}$, and $|C|=3.75 \times 10^{-4}\left|H_{b}\right|$ are depicted with dashed curves. Parameters of calculations are borrowed from Ref. [30]: $\lambda=715 \mathrm{~nm}, R_{p}=35 \mathrm{~nm}, \varepsilon_{p}=2$, $u=R_{p}, \varepsilon_{a}=1, \varepsilon_{b}=10^{2}, \varepsilon_{o}=-1.06+0.288 i, \varepsilon_{e}=8.09+1.06 i$, and $h=1600 \mathrm{~nm}$.

is the amplitude transmission coefficient, and

$$
H_{\text {inc }}\left(k_{x}\right)=\frac{1}{2 \pi} \int_{-\infty}^{\infty} H_{i n c}\left(x^{\prime}\right) \mathrm{e}^{-i k_{x} x^{\prime}} d x^{\prime}
$$

is the incident field spectrum calculated using the inverse Fourier transform. Impedances of the input $\gamma_{a}=\frac{1}{\varepsilon_{a}} \sqrt{\varepsilon_{a}-\frac{k_{x}^{2}}{k_{0}^{2}}}$ and output $\gamma_{b}=\frac{1}{\varepsilon_{b}} \sqrt{\varepsilon_{b}-\frac{k_{x}^{2}}{k_{0}^{2}}}$ media as well as the longitudinal wavenumber $k_{z}=\sqrt{k_{0}^{2} \varepsilon_{o}-k_{x}^{2} \varepsilon_{o} / \varepsilon_{e}}$ should be inserted into the definition of transmission coefficient (4), where $k_{0}=\omega / c$ is the vacuum wavenumber. Small transmission $|t(0)|^{2} \sim \mathrm{e}^{-2 h \text { Rekz }}$ of the background normally incident plane wave with $k_{x}=0$ confirms the conclusion made from the dispersion diagram in Figure 1b: the background field exciting the dipoles is not transmitted. Using Eqs.(3)-(5) and the theory provided by Section S1 of Supporting Information we calculate transmitted fields and expose them in Figures 3-6 as lines marked "calculation". The algorithm for numerical calculation of the transmitted field is as follows.

1. Discretize the incident field in the range of coordinates from $x_{1}=-X$ to $x_{N}=+X$ as $H_{\text {inc }}\left(x_{i}\right)$, where $i=1, \ldots, N$ and $x_{i}=x_{1}+(i-1)\left(x_{N}-x_{1}\right) / N$. Calculate numerically Eq. (5) using the inverse Fast Fourier Transform (FFT). The resulting discrete spectrum is $H_{i n c}(i)$.

2. Evaluate the transmission coefficient (4) at the discrete points $i=1, \ldots, N$. Wavenumbers corresponding to $i$ are equal to $k_{x}(i)=(i-1) \pi / X$. Due to the finite value of $X$ we need to employ the numerical version of transmission coefficient in the form $\sum_{j=-\infty}^{\infty} t\left(k_{x}(i)+j \pi N / X\right)$. In our case, it is sufficient to keep only two terms $t\left(k_{x}(i)\right)+t\left(k_{x}(i)-\pi N / X\right)$ describing the plane waves with $k_{x} \geq 0$ and $k_{x}<0$, respectively.

3. Compute Eq. (3) using the FFT again.

We specify the parameters of calculation as in Ref. [30]: wavelength $\lambda=715 \mathrm{~nm}$, permittivities of the input $\varepsilon_{a}=1$ and out- put $\varepsilon_{b}=10^{2}$ media, permittivities and thicknesses of respectively metal $\varepsilon_{m}=(0.14+i 2.06)^{2}$ and $h_{m}=10 \mathrm{~nm}$ and dielectric (silica) $\varepsilon_{d}=1.45^{2}$ and $h_{d}=10 \mathrm{~nm}$ layers, and number of unit cells $N=$ 80 (effective permittivities of the type-II HMM $\varepsilon_{o}=-1.06+$ $0.288 i$ and $\varepsilon_{e}=8.09+1.06 i$, its thickness $\left.h=1600 \mathrm{~nm}\right)$. The value of $\varepsilon_{b}$ is artificially large to save as much propagating waves at the output from HMM as possible, although a smaller value $\varepsilon_{b}=3.5^{2}$ is also suitable (see Section S1.5 of Supporting information). Albeit we demonstrate the approach for non-realistic material parameters of the metal in this article, the realistic parameters could be also exploited. In Section S1.5, one may find the similar improvement of the contrast function for silver at several wavelengths.

Now we derive a physically insightful simplified theory having the accuracy close to that of the rigorous calculations. In figures we refer it as "theory". We start with transmission of the radiation of a single dipole assuming $H_{i n c}(x)=H_{d}(x)$. We impose the following restrictions. First, the losses of the hyperbolic metamaterial are negligible $\left(\varepsilon^{\prime \prime} \ll \varepsilon^{\prime}\right)$. Second, the thickness of the HMM slab is as small as $\left|k_{z}\right| h \ll 1$. Third, the transmission through HMM-II changes mainly near $\pm k_{0} \sqrt{\varepsilon_{e}^{\prime}}$ as shown in Figure 3a providing a pair of images due to positive and negative wavenumbers (right and left images in Figure 1a). The zero-transmission zone is related to the low-k waves gap in Figure 1b. We will further consider only a half of the spectrum assuming $k_{x}=+k_{0} \sqrt{\varepsilon_{e}^{\prime}}+\kappa$, where $\kappa \ll k_{0} \sqrt{\varepsilon_{e}^{\prime}}$. Fourth, the shape of the input dipolar signal is modeled by the Gaussian profile as $H_{d}(x)=A_{0} \exp \left(-x^{2} / 2 \Delta x^{2}\right)$, where $\Delta x$ is the beam half-width at the intensity level $\left|H_{d}\right|^{2} / e, e$ is the base of the natural logarithm, and $A_{0}$ is the complex amplitude. Then the inverse Fourier transform yields the signal spectrum $H_{d}\left(k_{x}\right)=\frac{A_{0} \Delta x}{\sqrt{2 \pi}} \exp \left(-k_{x}^{2} \Delta x^{2} / 2\right)$. The complex-valued halfwidth $\Delta x$ models the phase of the dipole magnetic field as it is shown in Figure 3b.

Having all these simplifications in mind and using small parameters $\kappa$ and $\varepsilon_{a} / \varepsilon_{b} \ll \varepsilon_{e}^{\prime} / \varepsilon_{b} \ll 1$, we are able to take a closedform integral (3) over the positive wavenumbers. Eventually, we get the transmitted magnetic field of the dipole radiation as 

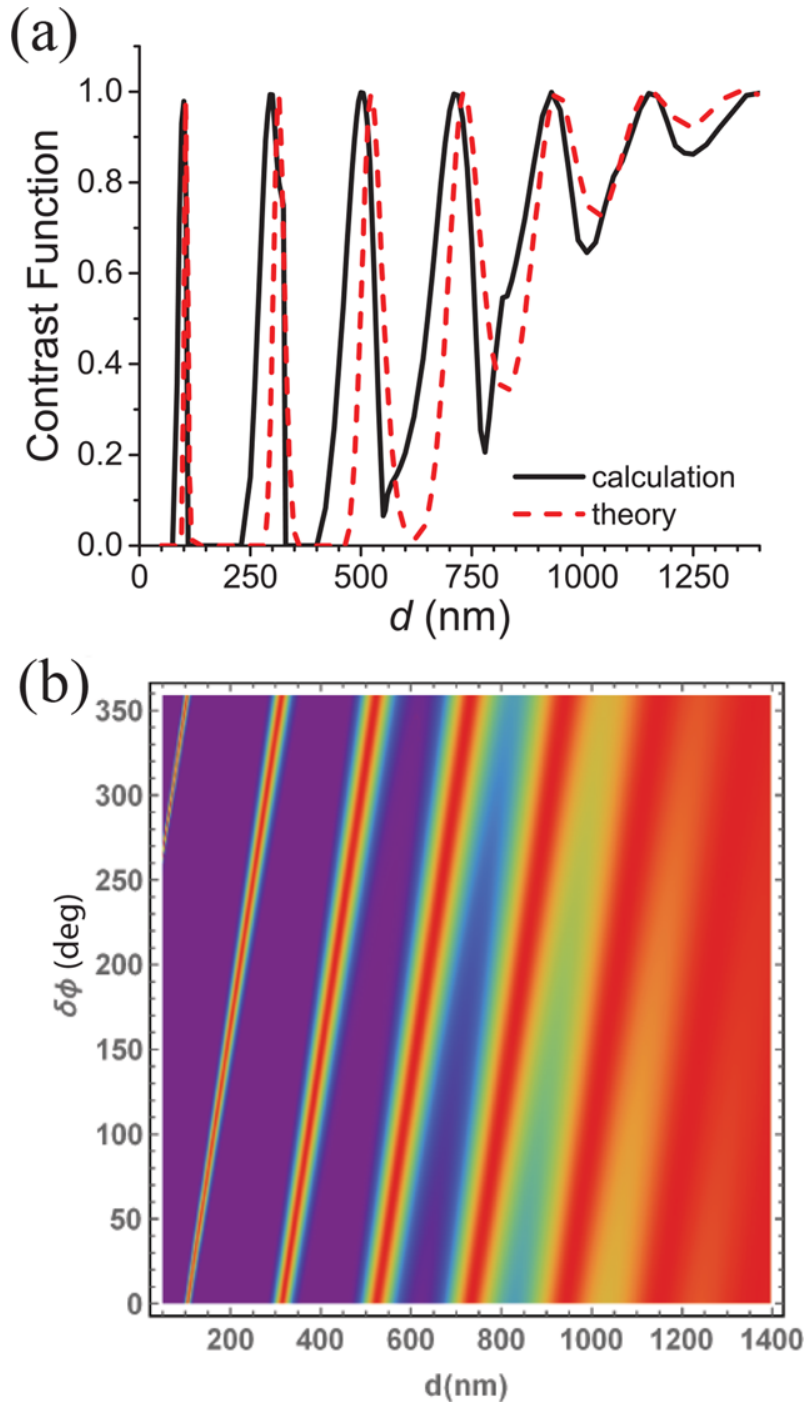

Figure 4. Contrast function against distance $d$ and phase difference $\delta \phi$ for two dipoles illuminated by the background field of plane wave. a) Comparison of the results of calculation and simplified theory reveals good coincidence. b) Dependence of the contrast function on the distance between objects $d$ and phase shift $\delta \phi$ obtained with the help of the simplified theory. Purple (red) color corresponds to $C F=0(C F=1)$. Parameters of calculation and retrieved parameters of the simplified theory are given in the caption of Figure 3.

follows (the details are given in Section S3 of Supporting Information)

$H_{t r}(x)=C \exp \left(-\frac{(x-b)^{2}}{2 a^{2}}\right) \mathrm{e}^{i k_{0} \sqrt{\varepsilon_{e}^{\prime}} x}$,

where $a>\operatorname{Re}(\Delta x)$ is the half-width of the transmitted field, $b=$ $b^{\prime}+i b^{\prime \prime}$ and $C$ are complex parameters. Real part $b^{\prime}$ of complex parameter $b$ is responsible for the image displacement along the axis $x$.

Since the background field is terminated by type-II HMMs, the coherent superposition of the fields of two identical dipoles at distance $d$ reads

$$
\begin{aligned}
H_{t r}(x)= & C \mathrm{e}^{i k_{0} \sqrt{\varepsilon_{e}^{\prime}} x}\left[\exp \left(-\frac{(x-b)^{2}}{2 a^{2}}\right)\right. \\
& \left.+\sigma \mathrm{e}^{-i k_{0} \sqrt{\varepsilon_{e}^{\prime}} d} \exp \left(-\frac{(x-b-d)^{2}}{2 a^{2}}\right)\right],
\end{aligned}
$$

where $\sigma=|\sigma| \exp (\delta \phi)$ takes into account the difference in excitation of the dipoles. Further we assume that the excitation differs only by phase shift $\delta \phi$, that is $|\sigma|=1$ (the influence of $|\sigma|$ is demonstrated by Figure S5 in Supporting Information). Quantity $\left|H_{t r}(x)\right|^{2}$ proportional to the intensity now can be straightforwardly calculated. Distinguishing two transmitted signals (peaks) requires the deep dip between them. Accordingly, the intensity at the midway $x_{m w}=b^{\prime}+d / 2$ between two peaks

$$
\begin{aligned}
\left|H_{t r}\left(x_{m w}\right)\right|^{2}= & 2|C|^{2} \exp \left(\frac{b^{\prime \prime 2}-(d / 2)^{2}}{a^{2}}\right) \\
& \times\left[1+\cos \left(k_{0} \sqrt{\varepsilon_{e}^{\prime}} d+\frac{b^{\prime \prime} d}{a^{2}}-\delta \phi\right)\right]
\end{aligned}
$$

can provide the ideal dip $\left|H_{t r}\left(b^{\prime}+d / 2\right)\right|^{2}=0$, if the phase equals

$\left(k_{0} \sqrt{\varepsilon_{e}^{\prime}}+b^{\prime \prime} / a^{2}\right) d-\delta \phi=\pi+2 \pi m$,

where $m$ is an integer number. For $\delta \phi=0$, this condition of destructive interference of two individual beams from the sources separated by distance $d$ holds true only for specific values of $d$. Therefore, the contrast function is a periodical function of the distance $d$. When $d$ is great, the intensity at the $\operatorname{dip} \mid H_{t r}\left(b^{\prime}+\right.$ $d / 2)\left.\right|^{2} \sim \exp \left(-d^{2} / 4 a^{2}\right)$ is always a small value and CF is high: two sources are distinguishable. If one is able to produce a proper phase difference $\delta \phi$ between two signals at each $d$, the contrast function will be always perfect $(C F=1)$, even in the deep subwavelength regime.

Intensity $\left|H_{t r}(x)\right|^{2}$ depends on 4 real parameters: $|C|, a, b^{\prime}$, and $b^{\prime \prime}$. They can be calculated within the restrictions discussed above, but then they have nothing to do with realistic systems. Instead, we will show that these 4 parameters are sufficient to well describe a regular (lossy and thick) hyperlens. Parameters $|C|$, $a$, and $b^{\prime}$ have a clear meaning and, therefore, can be straightforwardly retrieved from the intensity profile of the dipole radiation transmitted by a lossy and thick hyperlens. Such calculated profile is shown as a solid curve in Figure 3c. Its peak intensity, half-width, and displacement equal respectively $\left|H_{p}\right|^{2} /\left|H_{b}\right|^{2}=$ $1.3 \times 10^{-6}, a=283.2 \mathrm{~nm}$, and $b^{\prime}=888.7 \mathrm{~nm}$. Albeit parameter $b^{\prime \prime}$ could be found from the phase of the transmitted signal, we will use Eq. (9) instead. We determine $b^{\prime \prime}$ estimating the slope of the straight line $\delta \phi(d)$, at which $C F=1$, plotted for the thick and lossy hyperlens in the inset of Figure 3c. Value $b^{\prime \prime}=421.5$ $n m$ allows finding $|C|=\exp \left(-b^{\prime \prime 2} / 2 a^{2}\right)\left|H_{p}\right|=3.75 \times 10^{-4}\left|H_{b}\right|$. Retrieved parameters introduced into Eqs. (7) and (9) result in the similar transmission as exact one (see dashed vs. solid curve in Figure 3c).

Although the simplified theory does not catch the asymmetry of intensity profiles, it exhibits the correct dependence on distance $d$ and phase difference $\delta \phi$. In fact, there exists a remarkably 
(a)
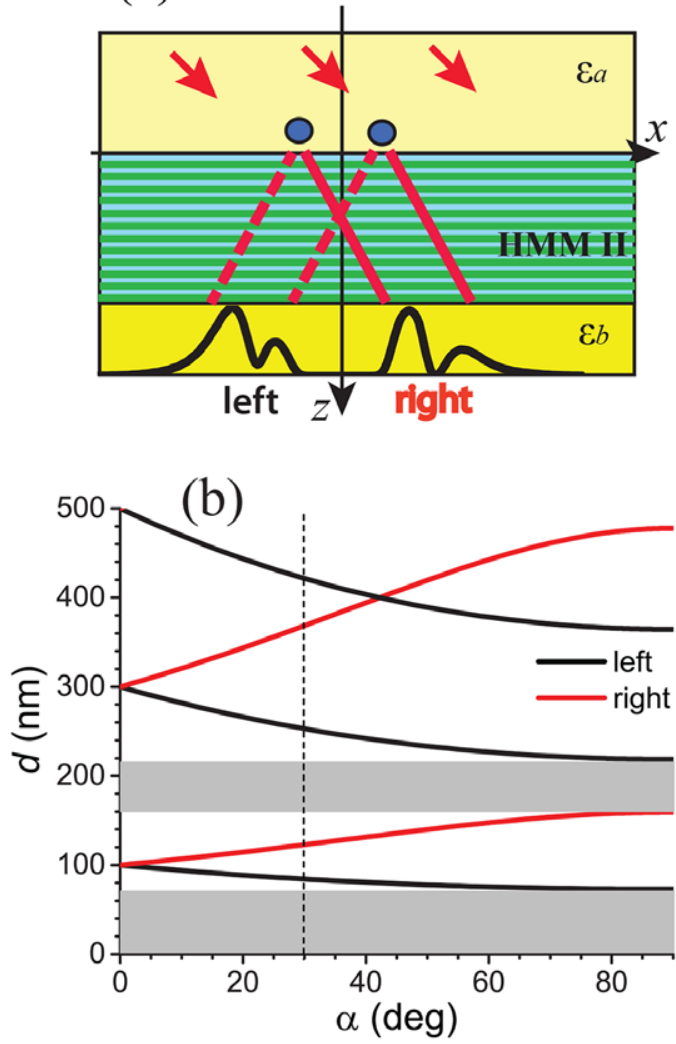
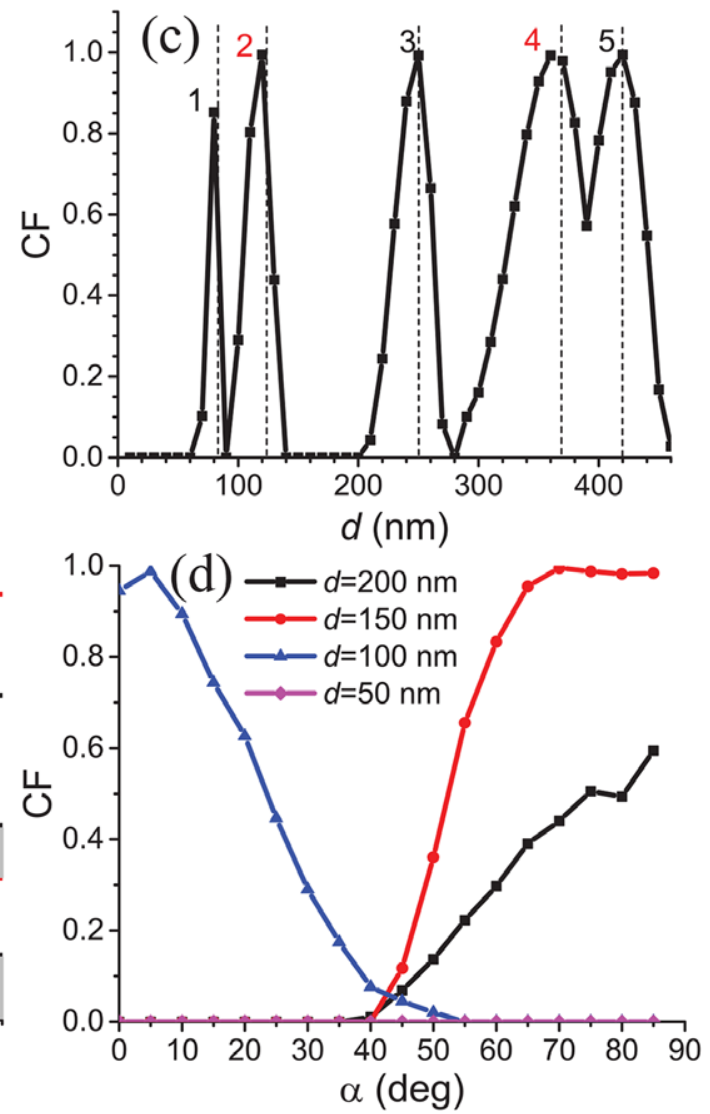
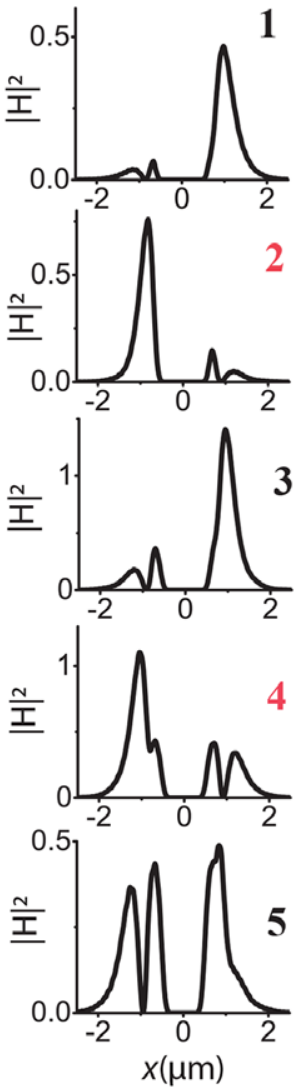

Figure 5. Obliquely incident plane waves exhibit the phase shift for improving the contrast function. a) Asymmetrically transmitted waves under the oblique incidence. b) Lines of C F $=1$ defined by Eq. (10). Vertical dashed line is positioned at $\alpha=30^{\circ}$. c) Calculated contrast function vs. distance between sources $d$ at the angle of incidence $\alpha=30^{\circ}$. Transmitted intensities $|H|^{2}$ (in a.u.) at the maximums 1-5 are depicted on the right-hand side of the figure. Vertical dashed lines correspond to $C F=1$ according to Eq. (10) following from the simplified theory. d) Calculated contrast function vs. angle of incidence $\alpha$ for various values of $d$. Parameters of the system are mentioned in the caption of Figure 3.

nice correspondence between the peaks and dips in oscillatory dependence $C F(d)$ shown in Figure 4a. Positions of the peaks almost perfectly match. Discrepancies in the dips can be related to the involved intensity profiles which influence more when the contrast is low. Nevertheless, the magnitude of oscillations in both calculations is agreed. The simplified theory provides the way of very fast prediction of the phase shifts $\delta \phi$ corresponding to the perfect contrast function $C F=1$. The diagram in Figure $4 \mathrm{~b}$ clearly demonstrates that there is the range of phase differences $\delta \phi$ providing high $C F$ s for each distance $d$. This range is wider for greater $d$. Eventually, when the sources are well separated we arrive at the phase-independent high contrast imaging.

It should be stressed that the four parameters the theory relies on can be found also from simulations or experimental data. These parameters characterize the system "single source + hyperlens" and do not require the distance between sources. Finding four effective parameters recalls the procedure of the retrieval of material parameters well adopted in the field of metamaterials. We believe that the theory should not depend much on the incident radiation, but it is rather determined by the transmission properties of the HMM-II. Generally speaking, the theory can be extended to the type-I hyperbolic and elliptic slabs (see Sections S4 and S5 in Supporting Information).

\section{Practical Designs to Introduce the Phase Difference}

The easy way to get the required local phase difference is the oblique incidence of a plane wave (see Figure 5a). Unlike the normal incidence, the transmitted pattern is asymmetric now. Further we will refer to the left- and right-hand side patterns. Assuming angle of incidence $\alpha$ we write the phase difference $\delta \phi=2 \pi \sqrt{\varepsilon_{a}} d \sin \alpha / \lambda$. It depends on $d$, therefore, the close sources cannot acquire large phase shifts. Introducing this phase shift into the condition of the unity contrast function Eq. (9), one writes

$d(\alpha)=\frac{\pi+2 \pi m}{\left(\sqrt{\varepsilon_{e}^{\prime}}-\sqrt{\varepsilon_{a}} \sin \alpha\right) 2 \pi / \lambda+b^{\prime \prime} / a^{2}}$.

Exploiting the same parameters of the type-II hyperbolic metamaterial as before, water $\left(\varepsilon_{a}=1.33^{2}\right)$ as the input and germanium $\left(\varepsilon_{b}=4^{2}\right)$ as the output medium, and silicon $\left(\varepsilon_{p}=3.5^{2}\right)$ beads of radius $R=35 \mathrm{~nm}$ as scatterers, in Figure $5 \mathrm{~b}$ we plot the dependencies $d(\alpha)$ according to Eq. (10). The contrast function takes value 1 either for the right-hand (red curves) or left-hand (black curves) transmitted rays. Because of 


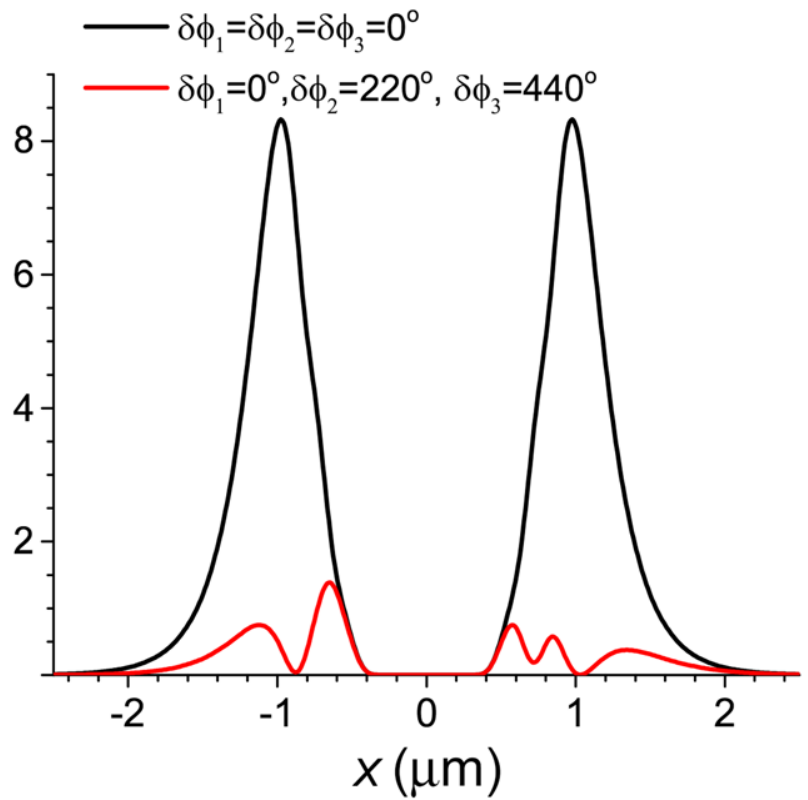

Figure 6. Intensity transmitted through the dark-field hyperlens to observe three equivalent dipoles in a row illuminated by a normally incident wave and possessing phases $\phi_{1}, \phi_{2}$, and $\phi_{3}$. The distance between the dipoles 1 and 2 and 2 and 3 equals $200 \mathrm{~nm}$. Parameters of the system are provided in Section 2. Parameters of the hyperlens are the same as in the caption of Figure 3.

the periodicity the dependence $d(\alpha)$ reminds the band diagram. Notice that the objects separated by some distances $d$ cannot be perfectly resolved at any angle $\alpha$ (see the grey-colored gaps in Figure 5b). A gap for small $d$ exhibits the limitation of the particular technique to create the sufficient phase difference. For great distances $d$ the contrast function $C F=1$ can be achieved for several values of $\alpha$. At $\alpha=30^{\circ}$ one can observe 5 values of $d$, where $C F=1$. These values are shown in Figure $5 c$ with the vertical dashed lines. They almost coincide with the maximums following from the calculation (we choose the greatest contrast function no matter, if it is from the left- or right-hand side). Several profiles $1-5$ of the transmitted signal demonstrate that the close to unity contrast function is indeed realized either from the left- or right-hand side. If one wants to employ the single side, angle of incidence $\alpha$ should flip the sign, i.e. one should change the direction of illumination. When the objects are close to each other, the transmitted high-contrast signal is weak due to the destructive interference (profile 1). Well separated objects may be resolved using both right- and left-hand side rays. Figure $5 \mathrm{~d}$ demonstrates that for distance $d=50 \mathrm{~nm}$, which is in the gap in Figure 5b, the objects cannot be resolved at all, but for the gap around $d=200 \mathrm{~nm}$ the contrast function 0.5-0.6 is feasible. The objects at distance $d=100 \mathrm{~nm}$ can be well resolved without a phase difference even with normally incident plane waves.

To characterize the contrast function we use spherical scattering objects. However, all conlusions can be extended for cylindrical scattering objects as well. As it can be observed in Figure S2 in Supporting Information, the perfect contrast function maintains for distances $d=100 \mathrm{~nm}, d=300 \mathrm{~nm}$, and $d=500 \mathrm{~nm}$ as pre- dicted by Figure 5b. Thus, the form of a scatterer does not really matter.

To overcome the limitations of the proposed scheme with inclined plane wave illumination, one may think of introducing required $\delta \phi$ by a properly designed metasurface. This idea is inspired by the recent progress in metasurface-based phase manipulation. ${ }^{[33-37]}$ In the classical design ${ }^{[38,39]}$ the $2 \pi$ phase shift is produced by a unit cell with the properly made $\mathrm{V}$-shaped metallic antennas, the phase difference between the nearest neighbor antennas being equal to $45^{\circ}$. If one intends to resolve two beads separated by distance $d$, each of them should be illuminated by light from its own antenna. Then the dimensions of individual antennas will define the maximum distance $d$. Scaling down the dimensions from $10 \mu \mathrm{m}$ wavelength and $2 \mu \mathrm{m}$ antenna's dimension ${ }^{[38]}$ to respectively $715 \mathrm{~nm}$ and $150 \mathrm{~nm}$ in the current research, it could be possible to resolve nanoobjects separated by the distance $d \sim 150 \mathrm{~nm}$. Since the metal-containing metasurfaces have rather poor performance in transmission, it could be better to exploit an all-dielectric metasurface like in Ref. [40]

Another design of a metasurface applicable for our purposes has been recently proposed in Ref. [41] It consists of a periodic lattice of subwavelength Fabry-Pérot resonators formed by the perfectly reflecting (distributed Bragg reflector) and transmitting mirrors. The elements of the metasurface (pixels) can be controlled individually creating the same signal amplitude and required phase. As indicated in Ref. [41] the device keeps its functionality even for a single pixel. If we put such a metasurface on top of the scatterers, it would be feasible to have an arbitrary phase difference $\delta \phi$ limited by the size of the pixel only.

\section{Discussion and Conclusion}

More than two scatterers can be resolved using the phase-shift technique, too. However, in this case the closed form calculations of the contrast function are not straightforward. The contrast function itself should be redefined, e.g., as a multibranch function, with each of the branches regarded any two radiating dipoles. To find the phases that should be imposed on the dipoles one needs to perform optimization and make all contrast functions as large as possible simultaneously. Figure 6 shows the possibility in principle to resolve three dipoles in a row. Without an extra phase shift the images of the sources are indistinguishable. But the phase differences between adjacent dipoles make them visible, though the contrast functions are not perfect. Thus, the phase mask on top of a sample can help in visualizing deep subwavelength features of a scatterer. In some sense it recalls coherent phase-contrast microscopy ${ }^{[9]}$ intended to determine the map of sample thicknesses using information about the phases. It uses indirect visualization owing to the correspondence between the thickness and the acquired phase. Here, however, we employ the inverse procedure to directly observe the sample using the phase mask.

Our analysis is not fixed with the certain parameters of hyperlens and scattering objects. Any type-II hyperbolic metamaterial slab could be exploited as a dark-field hyperlens (see Section S1.5 of Supporting Information). To improve the performance of the lens, it should be optimized with respect to the losses. The subwavelength features of the sample serve as the scattering 
objects. In subwavelength regime they can be treated as electrical dipoles. We consider the spherical objects here only to specify the polarizability, because it is well known for a sphere.

There are two important issues that should be mentioned, but cannot be discussed in details in this article. The first issue is the choice of the phase mask (the way to introduce the phase difference). To be applicable for any subwavelength object, the phase mask should take the advantages of the tunable metasurface, e.g., proposed in Ref. [41] Dynamically changing the phase shifts provided by the metasurface pixels we would arrive at the optimal mask with respect to the phase contrast. The second problem that may arise for the imaging of a realistic subwavelength object is that each point of the dipole will be mapped into a circle (the rays diverge in all directions) and, therefore, the focusing system is needed after the dark-field hyperlens. The focusing system can be made as an additional multilayer that converges rays being at the same time type-II HMM (similar idea has been recently exploited in Ref. [42]).

To conclude, we have elaborated the technique to perfectly visualize subwavelength features of a scattering object after the dark-field hyperlens imaging. It is based on introduction of a phase difference between the scatterers, which improves a contrast function up to unity. The effect stems from destructive interference of the output signals of individual scatterers enhancing the dip between two imaged scatterers. To optimize the contrast function we have developed a simplified theory and validated its functioning for planar and cylindrical hyperlenses. We have demonstrated how the phase difference can be achieved using obliquely incident light. An alternative approach is to illuminate the objects through the phase-shifting metasurfaces. Such a technique can help to distinguish a couple of subwavelength features in a massive transparent object. Since the technique can be extended to the case of multiple scatterers, we look forward seeing further application of this kind of dark-field phase microscopy soon.

\section{Supporting Information}

Supporting Information is available from the Wiley Online Library or from the author.

\section{Acknowledgements}

We acknowledge Villum Fonden (DarkSILD project) for financial support.

\section{Conflict of Interest}

The authors declare no conflict of interest

\section{Keywords}

hyperlens, metamaterials, superresolution

Received: August 7, 2017

Revised: November 3, 2017

Published online: January 11, 2018
[1] M. Born, E. Wolf, Principles of Optics, Cambridge University Press, Cambridge 1997.

[2] J. B. Pendry, Phys. Rev. Lett. 2000, 85, 3966.

[3] F. Zernike, Physica 1942, 9, 686.

[4] F. H. Smith, Research (London) 1955, 8, 385.

[5] G. Nomarski, J. Phys. Radium 1955, 16, 9 S.

[6] R. Hoffman, L. Gross, Appl. Opt. 1975, 14, 1169.

[7] G. Popescu, T. Ikeda, R. R. Dasari, M. S. Feld, Opt. Lett. 2006, 31, 775.

[8] M. Kemmler, M. Fratz, D. Giel, N. Saum, A. Brandenburg, C. Hoffmann, J. of Biomedical Optics. 2007, 12, 064002.

[9] C. Joo, T. Akkin, B. Cense, B. H. Park, J. F. de Boer, Opt. Lett. 2005, $30,2131$.

[10] J. L. Nadeau, Y. B. Cho, C. A. Lindensmith, Opt. Lett. 2015, 40, 4114.

[11] Z. Jacob, L. V. Alekseyev, E. Narimanov, Opt. Express 2006, 14, 8247.

[12] Z. Liu, H. Lee, Y. Xiong, C. Sun, X. Zhang, Science 2007, 315, 1686.

[13] I. I. Smolyaninov, Y.-J. Hung, C. C. Davis, Science 2007, 315, 1699.

[14] J. Sun, M. I. Shalaev, N. M. Litchinitser, Nature Commun. 2015, 6, 7201.

[15] D. R. Smith, D. Schurig, Phys. Rev. Lett. 2003, 90, 077405.

[16] A. Poddubny, I. Iorsh, P. Belov, Y. Kivshar, Nature Photon. 2013, 7, 948.

[17] P. Shekhar, J. Atkinson, Z. Jacob, Nano Convergence 2014, 1, 14.

[18] L. Ferraria, C. Wub, D. Lepaged, X. Zhangb, Z. Liu, Progress in Quantum Electronics 2015, 40, 1.

[19] Y.-C. Chang, C.-H. Liu, C.-H. Liu, S. Zhang, S. R. Marder, E. E. Narimanov, Z. Zhong, T. B. Norris, Nature Ccommun. 2015, 7, 10568.

[20] R. Chandrasekar, Z. Wang, X. Meng, A. Lagutchev, Y. L. Kim, A. Wei, A. Boltasseva, V. M. Shalaev, Proceeding of the Conference on Lasers and Electro-Optics, San Jose, USA (OSA 2016), JTh4A.4.

[21] S. S. Kruk, Z. J. Wong, E. Pshenay-Severin, K. O'Brien, D. N. Neshev, Y. S. Kivshar, X. Zhang, Nature Commun. 2016, 7, 11329.

[22] A. N. Poddubny, I. V. Iorsh, A. A. Sukhorukov, Phys. Rev. Lett. 2016, $117,123901$.

[23] J. Sun, T. Xu, N. M. Litchinitser, Nano Lett. 2016, 16, 7905.

[24] J. S. T. Smalley, F. Vallini, S. A. Montoya, L. Ferrari, S. Shahin, C. T. Riley, B. Kanté, E. E. Fullerton, Z. Liu, Y. Fainman, Nature Commun. 2017, 8, 13793.

[25] J. Benedicto, E. Centeno, R. Pollès, A. Moreau, Phys. Rev. B 2013, 88, 245138.

[26] T. Xu, A. Agrawal, M. Abashin, K. J. Chau, H. J. Lezec, Nature 2013, 497, 470.

[27] R. Maas, E. Verhagen, J. Parsons, A. Polman, ACS Photonics 2014, 1, 670.

[28] E. Centeno, A. Moreau, Phys. Rev. B 2015, 92, 045404.

[29] R. Maas, J. van de Groep, A. Polman, Optica 2016, 3, 592.

[30] T. Repän, A. V. Lavrinenko, S. V. Zhukovsky, Opt. Express 2015, 23, 25350.

[31] M. D. Levenson, N. S. Vishvanathan, R. A. Simpson, IEEE Transaction on Electron Devices 1982, ED-29, 1828.

[32] L. Novotny, B. Hecht, Principles of Nano-Optics, Cambridge University Press, Cambridge 2012, Ch. 4.

[33] N. Yu, F. Capasso, Nature Materials 2014, 13, 139.

[34] A. E. Minovich, A. E. Miroshnichenko, A. Y. Bykov, T. V. Murzina, D. N. Neshev, Y. S. Kivshar, Laser Photon. Rev. 2015, 9, 195.

[35] X. Zhu, C. Vannahme, E. Højlund-Nielsen, N. A. Mortensen, A. Kristensen, Nature Nanotechnology 2016, 11, 325.

[36] H.-T. Chen, A. J. Taylor, N. Yu, Rep. Prog. Phys. 2016, 79, 076401.

[37] S. B. Glybovski, S. A. Tretyakov, P. A. Belov, Y. S. Kivshar, C. R. Simovski, Phys. Rep. 2016, 634, 1. 
[38] N. Yu, P. Genevet, M. A. Kats, F. Aieta, J.-P. Tetienne, F. Capasso, Z. Gaburro, Science 2011, 334, 333.

[39] X. Ni, N. K. Emani, A. V. Kildishev, A. Boltasseva, V. M. Shalaev, Science 2011, 335, 427.
[40] Y. F. Yu, A. Y. Zhu, R. Paniagua-Domínguez, Y. H. Fu, B. Luk'yanchuk, A. I. Kuznetsov, Laser Photon. Rev. 2015, 9, 412.

[41] S. Colburn, A. Zhan, A. Majumdar, Sci. Rep. 2017, 7, 40174.

[42] L. Shen, H. Wang, R. Li, Z. Xu, H. Chen, Sci. Rep. 2017, 7, 6995. 